\title{
Etayage personnalisé et performances ciblées dans la formation à distance
}

\author{
Georges Ferone $^{1}$ et Didier Mendibil ${ }^{2}$ \\ ${ }^{1}$ Université Paris-Est, CIRCEFT-ESCOL (EA 4384), UPEC, 94010 Créteil Cedex, France \\ ${ }^{2}$ Université Paris-Est, UMR 8504, EHGO-Université Paris 1, France
}

\begin{abstract}
Résumé. Cette étude s'intéresse aux conséquences pédagogiques de la mise en œuvre d'enseignements à distance dans la formation professionnelle des adultes. L'étude de cas présentée montre que lorsqu'un enseignant propose un accompagnement basé sur la compréhension et la négociation des objectifs de travail, il favorise l'auto direction et la progression de ses étudiants. Elle permet aussi de préciser que cette réussite, au lieu d'être homogène, se produit avec une intensité et dans une temporalité qui varient selon les compétences considérées. Cela implique la nécessité pour l'enseignant d'expliciter les critères de réussite propres à la discipline qu'il enseigne pour sélectionner et ordonner ses objectifs selon la progression la plus favorable à l'amélioration de l'ensemble des performances de ses étudiants.
\end{abstract}

\section{Accompagnement à distance et auto direction de l'apprenant}

Avec le fort développement de la formation à distance (FAD), la question de l'efficacité de cette modalité de formation se pose avec plus d'acuité. Les chercheurs dans ce domaine s'intéressent en particulier à deux problématiques : celle de l'autonomie de l'étudiant, car la capacité de l'apprenant à diriger ses apprentissages est considérée comme déterminante pour sa réussite, et celle de la «présence à distance », car celle-ci est susceptible de rompre le sentiment d'isolement et de favoriser les apprentissages. Concernant l'autonomie, les recherches sur l'auto direction et sur l'auto régulation [1] ont pour " objectif d'expliquer comment l'apprenant réussit à se mettre au travail, à faire preuve de persévérance et à atteindre les buts fixés en contrôlant lui même ses processus d'apprentissage et sa motivation ». Pour Jézégou [2], l'auto direction de l'apprenant se caractérise par deux dynamiques en interaction, sa motivation et ses capacités d'auto régulation. Ainsi, plus l'étudiant choisit librement sa formation, plus il considère qu'elle lui permettra d'atteindre ses buts, plus il se sent efficace dans ses apprentissages et plus il sera motivé. La motivation est donc liée à la capacité de l'apprenant à réguler ses apprentissages. Zimmermann [3] décrit l'auto régulation en trois phases qui interagissent de manière cyclique : l'anticipation (analyse de la tâche), l'action (utilisation de stratégies) et l'autoréflexion (autoévaluation et jugement de l'action). Pour favoriser l'apprentissage autodirigé, Garrison [4] souligne le rôle pivot de la présence à distance qui se développe selon trois dimensions : la présence sociocognitive, socioaffective et pédagogique [5]. Dans notre étude, nous nous intéressons en particulier à la présence

This is an Open Access article distributed under the terms of the Creative Commons Attribution License 4.0, which permits unrestricted use, distribution, and reproduction in any medium, provided the original work is properly cited. 
pédagogique qui résulte « des interactions sociales que le formateur entretient à distance avec les apprenants pour soutenir les présences cognitive et socio-affective » [6].

$\mathrm{Si}$ ces différents travaux, brièvement présentés, constituent un socle de référence pour tout concepteur et animateur de formation, nous formulons deux remarques. La première concerne la présence du formateur : celle-ci est presque toujours étudiée dans un contexte de travail collaboratif où le rôle de l'enseignant consiste le plus souvent à favoriser les interactions sociales entre apprenants, quitte à s'effacer pour ne pas parasiter les échanges et pour mutualiser la construction des apprentissages. Cela suppose de pouvoir préciser, dans différentes situations, où commence ce "parasitage » et s'il tient aux registres de langage, au statut de formateur-évaluateur ou à d'autres formes d' "effet-maître » [7]. Mais, dans la majorité des dispositifs de formation à distance, dont celui que nous décrivons, le travail collaboratif n'est pas la modalité d'enseignement privilégiée. La seconde remarque concerne les apprenants : les recherches s'intéressent le plus souvent à la manière d'accroître la participation des étudiants, à leur motivation ou aux effets des interventions professorales sur le climat sociorelationnel [8] et il existe en définitive peu de travaux qui expliquent concrètement comment favoriser l'auto régulation des étudiants.

Nous avons donc choisi d'observer les effets de cette auto régulation sur l'aptitude à écrire des textes dans une discipline à la fois littéraire et scientifique et c'est pourquoi cet article vise à décrire de manière précise des modalités d'accompagnement susceptibles de développer des compétences en écriture de la géographie, de favoriser l'autorégulation des étudiants et d'en mesurer les effets sur les apprentissages attendus dans cette discipline.

\section{Comment favoriser l'auto régulation des étudiants ?}

L'étude de cas servant d'appui à notre réflexion a concerné un groupe de 50 étudiants inscrits à l'Université Paris-Est Créteil pour préparer, entièrement à distance, la totalité des unités d'enseignement de la première année du Master des métiers de l'enseignement du premier degré au cours de l'année universitaire 2012-2013. Il est important de préciser que la plupart de ces étudiants préparaient en même temps les épreuves du concours de recrutement de professeurs des écoles.

\subsection{Le contexte particulier de cette formation à distance}

On se souvient que la mise en œuvre d'une réforme de la formation des enseignants a entrainé une importante diminution du nombre des étudiants inscrits dans les Instituts Universitaire de Formation des Maîtres (IUFM). À la rentrée de septembre 2011 l'IUFM de Créteil qui disposait de moyens et de personnels pour le faire, a donc réagi à cette baisse d'effectifs en organisant une formation à distance au master d'enseignement du premier degré. C'est dans ce cadre nouveau que des enseignants ont dû s'interroger sur la manière d'adapter la formation à ces étudiants à distance. La préparation de cet article, élaborée en parallèle à la deuxième année de mise en œuvre de cette nouvelle formation, fut une des formes prises par cette réflexion didactique.

\subsubsection{Des conditions inhabituelles de communication}

La principale difficulté que rencontre l'enseignant en FAD est liée au manque de rétroactions immédiates ${ }^{1}$. En l'absence de réactions verbales et non verbales des apprenants, il est plus difficile de réguler la situation d'apprentissage. Cette régulation vise d'une part à installer un accord sur le niveau et la nature spécifique des contenus scientifiques qui doivent être maîtrisés par les étudiants

\footnotetext{
${ }^{1}$ Le recours à la classe virtuelle permet de résoudre en partie cette difficulté.
} 
et, d'autre part, sur les moyens d'en vérifier immédiatement la compréhension au moment même de leur énonciation par l'enseignant afin de limiter les malentendus ou les oublis. L'absence d'un contrôle auditif immédiat et global de la compréhension interdisant l'immédiateté des ajustements de réception et de compréhension, elle ouvre donc un large champ d'incertitude. Cette incertitude porte tout autant sur l'ensemble des éléments de la réception par les étudiants (acquisition et compréhension) des contenus de cours exposés que sur leur aptitude à les assimiler (niveau de lecture, mémorisation, conceptualisation) ou à les soumettre à la critique (questionnement, confrontation aux acquis ou aux représentations, démarche d'élucidation en cas d'ambiguïté) pour pouvoir les réutiliser de façon pertinente.

\subsubsection{Spécificité de la discipline géographique}

Dans le cadre de la formation initiale des enseignants du premier degré, la géographie constitue une matière scolaire apparemment bien connue puisque tous les étudiants ont été en contact avec elle au cours de leur scolarité primaire et secondaire. Mais elle relève d'une discipline universitaire dans laquelle peu d'étudiants ont effectivement poursuivi des études supérieures. On peut même considérer que c'est une discipline devenue trompeuse car relevant à la fois d'un « sens commun », que l'on croit bien partagé, et d'une discipline universitaire qui s'est récemment transformée au point de devenir méconnaissable pour tous ceux qui n'en ont pas suivi les dernières transformations. Celles-ci relèvent principalement de la transformation d'un paradigme naturaliste en un paradigme sociétal qui impose une rigueur accrue dans l'utilisation des termes et concepts relevant d'une construction scientifique ou issus du langage commun et de ses usages vernaculaires [9].

Pour expliquer cela, on évoquera simplement ici le fait que la discipline géographique, après avoir longtemps imité le modèle positiviste des sciences de la nature pour étudier les milieux naturels et les paysages, s'est dispersée en direction des sciences humaines et sociales au cours du dernier quart du $\mathrm{XX}^{\mathrm{e}}$ siècle et qu'elle semble, aujourd'hui, se recentrer sur quelques notions complexes aussi fondamentales que les territoires et l'environnement pour étudier la mondialisation [10]. Pour cette raison, l'enseignement de cette discipline « connue mais devenue incertaine » à un public hétérogène d'adultes rend nécessaire des ajustements de compréhension nombreux et différenciés selon l'âge et la scolarité des interlocuteurs. À cette difficulté s'ajoute celle d'avoir à utiliser les différents codes que mobilise usuellement la géographie (des textes, des images figuratives, des cartes, des données statistiques) qui ne sont pas simples à associer à distance.

Ces difficultés nous ont conduits à nous interroger sur la faisabilité d'un enseignement à distance de la géographie et sur les moyens de transformer sinon sa « directivité », du moins sa transmissivité. Deux voies principales d'amélioration s'ouvrent en effet pour l'enseignement à distance : se rapprocher des conditions de la communication orale directe par le perfectionnement des techniques de communication de l'image et du son, en temps réel ou différé ; ou bien renforcer et perfectionner les procédures d'accompagnement des cours écrits ou enregistrés pour en améliorer la réception. Sur cette seconde voie s'ouvre aussi un autre choix, non exclusif d'ailleurs, entre le renforcement d'une coopération qui favorise les interactions «transversales » entre étudiants, ou bien la diversification des formes de la collaboration « verticale » entre enseignant et apprenant. C'est dans cette dernière voie que s'est engagée notre réflexion car la distance rend difficile l'observation des interactions.

\subsection{L'étayage personnalisé de travaux de géographie réalisés en formation à distance}

\subsubsection{Les partis-pris de cette expérimentation}

La dimension collective du travail n'a pas été négligée puisque chaque thème étudié a donné lieu à l'élaboration de cours et de devoirs. Ces derniers ont été corrigés, commentés, formatés et, pour certains, publiés à destination d'un public collectif de référence : le groupe-classe. Ce sont les travaux les plus 
aboutis, le plus souvent améliorés par des ajouts du correcteur, qui ont été publiés sur la plateforme de travail du groupe. Toutefois, cette dimension collective du travail, proposée et mise en œuvre de façon systématique, n'a pas donné lieu à une évaluation de ses effets. Elle n'a pas non plus débouché sur la mise en œuvre et l'encouragement de procédures de travail collaboratif notamment en petits groupes. C'est plutôt sur le suivi du travail individuel et sur son accompagnement par l'enseignant qu'a porté l'effort d'évaluation et d'explicitation. Cela s'explique principalement par le fait que les contenus et les formes de l'enseignement de l'histoire et de la géographie ont été fortement orientés et conditionnés par la nature des épreuves du concours de recrutement (un devoir écrit du type dissertation en temps très limité) et donc par l'individualisation des objectifs à atteindre. C'est pourquoi l'enseignant a renforcé l'individualisation de son évaluation des travaux écrits et développé la personnalisation de ses conseils. On peut supposer que cette forme privilégiée de communication n'aura pas favorisé les coopérations, (ni empêché qu'elles ne se développent dans d'autres disciplines) mais les éléments permettant de l'affirmer avec certitude n'ont pas été recherchés ici.

\subsubsection{Le dispositif d'accompagnement}

Trois modalités de communication ont été choisies pour informer les étudiants des attentes institutionnelles et des critères d'évaluation retenus et pour informer l'enseignant des stratégies adoptées par les étudiants. L'enseignant a exposé dans un premier temps, l'organisation du cours, les travaux à réaliser et la méthodologie de travail. Celle-ci a été rappelée lors des corrections collectives modélisantes des six travaux proposés en géographie. Dans un deuxième temps, une correction individuelle de chaque devoir utilisant des critères spécifiques (voir ci-dessous) a été adressée aux étudiants. Enfin, pour préciser les stratégies des apprenants, il leur a été demandé de compléter un questionnaire d'enquête précisant, pour chaque devoir rendu, les conditions de sa réalisation (explication du choix du sujet et appréciation de son niveau de difficulté, temps consacré à la documentation puis à la rédaction, difficultés éprouvée pour différentes parties du devoir, etc.), les critères sur lesquels avaient porté leurs efforts (l'introduction et/ou la conclusion; le plan et la problématique ; les savoirs et les faits géographiques ; les notions, le vocabulaire spécifiques à la géographie ; la spatialisation ; les exemples précis illustrant des notions géographiques ; l'analyse des documents et leur intégration au devoir ; l'orthographe, le style et l'efficacité de l'écriture) et enfin, une évaluation commentée de la qualité des conseils donnés par l'enseignant lors de la correction du devoir précédent afin de mesurer leur réception par les étudiants (compréhension des critiques, adhésion aux conseils, désaccords éventuels). Contrairement aux deux précédents modes, qui procédaient principalement par apports de contenus et corrections d'erreurs, ce dernier mode d'échange, appuyé sur un « formulaire d'accompagnement » standardisé, permettait de pousser plus loin la personnalisation des demandes et des formulations de conseils pour éventuellement faciliter l'émergence d'un dialogue constructif. Son principal intérêt, à nos yeux, était de permettre aux étudiants d'évaluer plus précisément la qualité de leur travail, d'apprendre à en parler avec le formateurévaluateur et, par-là-même, d'adopter une méthodologie de travail plus performante parce que plus précisément orientée et plus lucidement choisie.

\subsubsection{Hypothèses et dispositif d'expérimentation}

Nous avons cherché à vérifier trois hypothèses : l'une, concernant l'amélioration des performances des étudiants, postule qu'elle passe par leur aptitude à mieux comprendre les objectifs de leur travail et les critères de sa réussite ; la suivante considère que cette aptitude peut être améliorée par la multiplication des échanges personnels avec l'enseignant; et la troisième envisage une efficacité différenciée de cette méthodologie selon la nature des performances considérées : on peut supposer, en effet, que toutes les compétences travaillées ne donnent pas lieu à des améliorations équivalentes selon qu'elles sont plus ou 
moins dépendantes des efforts du seul étudiant, des efforts du seul enseignant ou plutôt de l'intensité des relations établies entre eux.

L'étude, appuyée sur la correction de 115 devoirs écrits de géographie associés à 92 formulaires d'accompagnement remplis entre novembre 2012 et avril 2013, c'est-à-dire sur la totalité des travaux de géographie de cette «promotion » de formation à distance. Elle a principalement consisté à mesurer la progression de la qualité des travaux rédigés par les étudiants au regard de 7 critères différents d'analyse que le même enseignant à tous évalués sur une échelle de 0 à 5 et en répondant au jour le jour à toutes les demandes d'explicitation formulées dans les formulaires d'accompagnement des devoirs. Cette progression des résultats, considérée comme l'objectif visé n'a donc pas été analysée globalement mais, conformément à nos deux premières hypothèses de travail, en relation avec les échanges que les étudiants ont entretenus avec le formateur et, conformément à la troisième hypothèse, en examinant sur quelles compétences les étudiants ont progressé de façon significative et différenciée.

Pour prendre en compte les effets induits par les échanges personnalisés entre les étudiants et l'enseignant via les formulaires d'accompagnement, on a examiné si et comment les étudiants étaient entrés dans la communication collaborative proposée par l'enseignant. On a distingué trois profils différents d'étudiants selon leur niveau de communication. Ceux qui ont rempli tous les formulaires d'accompagnement de leurs devoirs et qui y ont formulé des questionnements qui se sont prolongés en des échanges auxquels l'enseignant a répondu par des conseils ciblés et personnalisés ont constitué le groupe « A » des 11 étudiants dits « communiquant». On peut considérer qu'ils ont été les plus grands bénéficiaires de l'accompagnement supplémentaire apporté par l'enseignant. A contrario, ceux qui n'ont pas rempli de formulaire et n'ont donc participé à aucun échange, ont constitué le groupe « $\mathrm{C} »$ des« 6 étudiants réputés «non communiquant». Entre ces deux extrêmes, on a considéré un groupe « $\mathrm{B}$ » de 14 étudiants dits «formalistes » car ils ont bien rendu des devoirs accompagnés de formulaires remplis mais ils l'ont fait sans questionner directement l'enseignant ni ouvrir les dialogues attendus par lui. Les autres étudiants inscrits qui, n'ayant rendu qu'un seul devoir dans l'année, ne pouvaient faire l'objet d'une évaluation de leur progression, n'ont donc pas été inclus dans un groupe.

Sur cette base, nous avons donc formulé l'hypothèse que les étudiants du groupe A obtiendraient des résultats en meilleure progression que ceux des étudiants du groupe B et a fortiori encore meilleurs que ceux des étudiants du groupe $\mathrm{C}$. Ayant aussi annoncé, en vertu de la troisième hypothèse, que les progrès des trois groupes ne porteraient pas sur les mêmes critères, nous avons pensé que ceux du groupe le plus communiquant (A) se porteraient plus vite sur les plus formels d'entre eux (cf. ci-dessous) et, plus significativement sur les plus disciplinaires d'entre eux (cf. ci-dessous) eu égard au surcroît d'aide personnalisée apportée par l'enseignant.

\subsection{Plus les étudiants communiquent, mieux ils semblent réussir}

Les résultats présentés semblent conformes aux attentes supposées par les deux premières hypothèses mais nous pensons que c'est la troisième hypothèse qui ouvre le plus de perspectives propices à de futures discussions et recherches.

\subsubsection{Les résultats des groupes selon leur niveau de communication}

Comme l'ont déjà montré de nombreuses recherches [11-13], les dispositifs d'accompagnement, le plus souvent mis en place pour aider les étudiants les plus fragiles, ratent leur cible et sont surtout investis par les meilleurs étudiants. Ce constat se vérifie dans notre étude puisque les étudiants qui réussissent le mieux ${ }^{2}$ sont ceux qui communiquent le plus. Ils constituent $42 \%$ de l'effectif du groupe A (52\% du groupe $\mathrm{B}$ et seulement $5 \%$ du groupe $\mathrm{C}$ ). Alors que les étudiants dont les notes sont inférieures à

\footnotetext{
${ }^{2}$ Les étudiants dont la moyenne générale aux examens de premier semestre est supérieure à 10/20.
} 
Relation entre la progression des résultats et le niveau des échanges avec l'enseignant

(chaque numéro représente un étudiant et chaque ellipse indique les 3 groupes distingués)

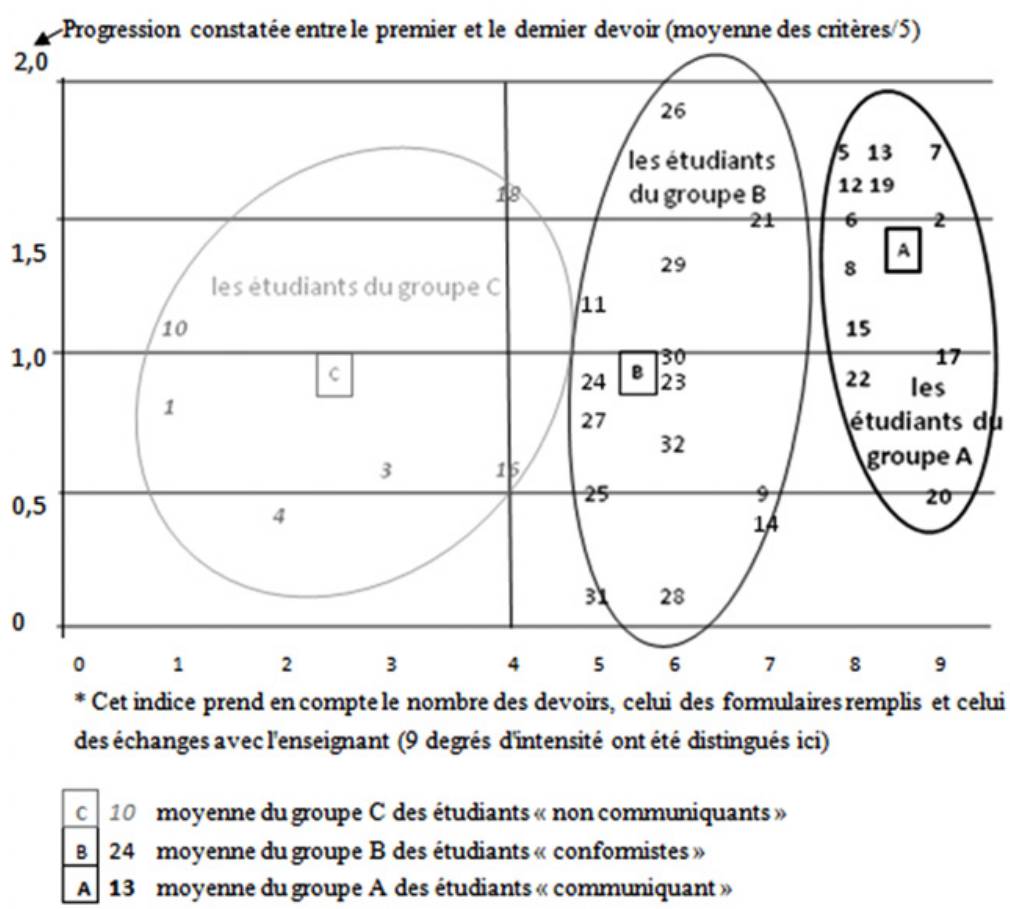

Figure 1. Relation entre la progression des résultats individuels et le niveau des échanges avec l'enseignant.

la moyenne à ces mêmes examens, constituent $22 \%$ du groupe $\mathrm{C}$ (groupe qui ne communique pas). Ils sont toutefois $22 \%$ à participer aux échanges (Groupe A). $56 \%$ des étudiants considérés comme les plus faibles appartiennent au groupe B.

Conformément à notre hypothèse, il existe bien une corrélation positive entre les résultats obtenus et le niveau des échanges entretenus avec l'enseignant. Car, si la moyenne des évaluations attribuées au milieu du premier semestre (notées ici sur 5) différenciait très peu les groupes $\mathrm{A}(1,83), \mathrm{B}(1,86)$ et $\mathrm{C}$ $(1,87)$, celle du second semestre a renversé le niveau de leurs performances puisque, calculée selon les même critères, elle était de 2,87 pour le groupe A, 2,74 pour le groupe B et 2,43 pour le groupe C.

Dans le détail de l'évolution des travaux individuels, que représente la Fig. 1, il faut d'abord observer la position occupée par chaque étudiant repéré par un numéro. En ordonnée ont été notées les progressions enregistrées entre le niveau du premier devoir et celui du dernier devoir corrigés de chaque étudiant. Les valeurs indiquées signalent les progressions constatées d'une note moyenne (sur 5) intégrant les 7 principaux critères d'évaluation retenus : une progression de 1,5 doit donc être considérée comme forte. L'axe des abscisses a été gradué de 1 à 9 pour indiquer différents niveaux d'échanges entre étudiants et enseignant : le niveau 1 correspond à deux devoirs rendus sans formulaire d'accompagnement ; le niveau 4 correspond à deux devoirs accompagnés de formulaires remplis mais sans amorce de dialogue ; le niveau 8 correspond à 4 devoirs accompagnés de formulaires et ayant donné lieu à au moins deux échanges suivis avec l'enseignant. On remarque que les plus fortes progressions (supérieures à 1,5 sur l'ensemble des critères) ont été bien plus nombreuses dans le groupe A des étudiants « communiquant» dont la progression moyenne a été de 1,3/5: trois fois plus que dans le groupe $\mathrm{B}$ et 6 fois plus que dans le groupe $\mathrm{C}$ des étudiants « non communiquant » dont la progression 
moyenne a été de $0,9 / 5$. L'inclinaison du nuage de points vers les valeurs basses du groupe «non communiquant », son étalement dans les valeurs moyennes et son élévation plus forte du côté des étudiants « communiquant » confirment le bien fondé de notre deuxième hypothèse mais la dispersion du nuage montre aussi que le critère de l'échange avec l'enseignant ne résume qu'une partie des dynamiques à l'œuvre. C'est sans doute pourquoi on a enregistré des cas de fortes progressions dans tous les groupes et c'est ce qui nous conduit à orienter maintenant notre propos différemment.

\subsubsection{La diversité des compétences travaillées}

L'un des aspects essentiels d'une formation à distance efficace consiste à développer l'autorégulation des étudiants en particulier par l'analyse des productions d'autrui et par l'exercice permanent de l'autoévaluation de leurs propres productions. L'acquisition de cette posture critique est complexe car elle suppose à la fois la maîtrise de connaissances dont l'acquisition demande un travail préalable d'apprentissage et une acculturation avec le positionnement scientifique d'une discipline particulière qui peut nécessiter des notions d'épistémologie. Mais à cela doit s'ajouter une posture métacognitive sur les manières de dire et faire la science ou d'en débattre, en particulier s'il s'agit d'argumenter des convictions personnelles. Parce qu'elles ne visent pas les mêmes objectifs et ne supposent pas les mêmes questionnements, ces différentes façons de travailler ne sont généralement pas enseignées en même temps. Pourtant, il semble bien nécessaire de les combiner, voire de les concilier dans la formation des enseignants.

C'est donc pour préparer les étudiants à ce type de positionnement qu'il a semblé important, notamment à un enseignant en géographie pour les raisons indiquées plus haut, de conduire un travail suivi de formation à la compréhension des critères multiples qui président à l'évaluation de la qualité d'une production écrite du type dissertation de concours d'accès à l'enseignement public. Le travail de correction des travaux d'étudiants s'est donc focalisé sur l'explicitation de ces critères et il a orienté le plus souvent possible les échanges personnalisés à propos des devoirs vers ces questions.

Toutefois, et cette considération revient à notre troisième hypothèse de travail, il n'est pas facile d'aborder une telle complexité, à distance, sans risquer de graves incompréhensions. Certains critères semblent plus accessibles au travail personnel de l'étudiant alors que d'autres supposent un étayage professoral ciblé ou une durée d'acculturation plus longue. Autrement dit, l'étayage professoral doit sérier et ordonner ses objectifs pour prétendre à un enseignement efficace. Si on résume les critères d'autoévaluation qui ont été explicités aux étudiants on constate qu'ils partagent la responsabilité de l'apprentissage. Il y a des compétences de nature formelle désignant des façons de faire, parfois scolaires, qui s'apprennent par l'imitation et la répétition des modèles validés par l'enseignement : une introduction, l'annonce d'un plan, une conclusion, le choix d'exemples illustratifs localisés en géographie, etc.. Ces procédures, souvent répétées, peuvent être facilement vérifiées par l'enseignant et donc acquises par les étudiants attentifs à ses remarques. Il y a des compétences de nature culturelle qui rassemblent tous les savoirs cognitifs, généraux ou spécialisés et tous les savoirs expressifs et linguistiques nécessaires à la communication. Elles relèvent principalement de la construction continue d'une culture personnelle dont les contours peuvent être flous mais dont les fondements communs constituent le cœur des civilisations. Elles relèvent donc de la responsabilité directe de l'étudiant et de son travail quotidien parfois orienté par celui des enseignants. Il y a enfin le domaine des compétences disciplinaires qui, parce qu'il demande la maîtrise d'un vocabulaire spécifique, la compréhension intime de notions réflexives complexes et la connivence avec des problématiques implicites, relève plus directement de la responsabilité de l'enseignant et demande nécessairement un temps plus long d'échanges avec les étudiants.

Cette typologie a conduit l'enseignant à observer vers quelles compétences se portaient les échanges et sur lesquelles s'observaient les plus notables progressions selon les groupes d'étudiants considérés. En première approximation, il lui semblait plus raisonnable d'espérer obtenir des résultats positifs rapides, 


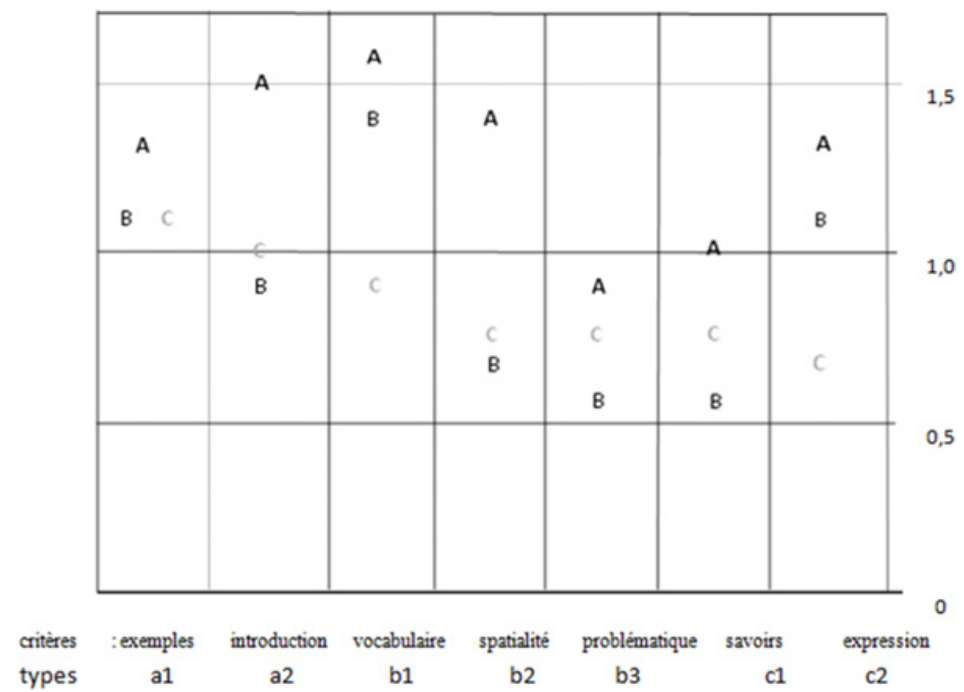

Figure 2. Progression des différents groupes au regard des différentes compétences travaillées.

et donc stimulants pour les étudiants, sur les compétences les plus formelles. L'usage des exemples localisés, simple illustration de faits géographiques, lui semblait être la compétence la plus aisément accessible à tous les étudiants. De même, l'élaboration d'une introduction convenablement structurée semblait pouvoir constituer l'objectif raisonnable des premiers efforts soutenus par l'ensemble du groupe. C'est pourquoi l'enseignant a placé ces deux compétences formelles aux premiers rangs de ses objectifs à atteindre.

Les compétences disciplinaires nécessitant plus de temps d'assimilation par des étudiants non spécialistes de la géographie mais aussi plus de temps de préparation de la part de l'enseignant elles ont été abordées dans un second temps. C'est d'abord la maîtrise du vocabulaire disciplinaire qui a été travaillée car l'approche de la spatialité et celle de la problématisation du plan d'exposition sont des aspects plus complexes qui demandaient un temps d'acculturation plus long. Ces questions ont fait l'objet de la plupart des échanges avec le professeur au second semestre. Enfin, les domaines du savoir et de l'expression qui semblaient d'abord relever de la responsabilité personnelle des étudiants ont été jugés moins accessibles aux stratégies de formation développées par l'enseignant. Cette appréciation de son potentiel d'efficacité a conduit le professeur à classer les 7 critères répertoriés dans la Fig. 2 dans l'ordre suivant d'opérationnalité supposée, en particulier dans ses relations avec les étudiants les plus « communiquant »: (a : compétences formelles, b : compétences disciplinaires, c : compétences culturelles) :

a1 - exemples, a 2 - introduction, b1 - vocabulaire, b2 - spatialité, b3 - problématique, c1 - savoirs, c2 - expression.

Il s'agit donc, au-delà des différences prévisibles des niveaux de performances atteints par les étudiants des différents groupes, d'observer si l'efficience de l'accompagnement professoral s'est bien vérifiée conformément à l'ordre indiqué ci-dessus.

\subsubsection{Des progrès différents selon les compétences considérées}

La Fig. 2 représente les progressions annuelles constatées pour chaque groupe d'étudiants sur l'ensemble des compétences travaillées, c'est-à-dire l'augmentation moyenne de la note (sur 5) attribuée en vertu de chacun des 7 critères d'évaluation entre le premier et le dernier devoir rendus. Elle confirme 
d'abord que les progressions n'ont pas été homogènes et qu'elles ont été très différentes selon les groupes considérés. Le «profil » du groupe A des étudiants « communiquant » montre une progression plus forte que les autres groupes et cela pour toutes les compétences considérées. Tous les groupes ont progressé d'au moins 0,5 point d'indice mais, pour les deux compétences a 2 et b2, la progression du groupe A a été plus forte de plus de $50 \%$.

Cela va donc dans le sens indiqué par nos deux premières hypothèses mais, en vertu de la troisième, nous nous attendions à ce que ce graphique fasse apparaître un parallélisme plus grand entre les « profils » des trois groupes : le résultat final imaginé aurait donc montré sur ce graphique 3 courbes, décalées mais parallèles, de $\mathrm{A}$, de $\mathrm{B}$ et de $\mathrm{C}$. Il aurait simplement indiqué que les groupes avaient progressé dans les mêmes directions mais avec des intensités différentes.

Deux aspects nous semblent donc très intéressants : d'une part les écarts constatés par rapport au «profil ordonné » appelé par notre hypothèse d'acquisition différenciée des compétences dans le temps ; d'autre part, les importants écarts du profil du groupe A par rapport aux profils des autres groupes. Le profil d'ensemble du groupe $\mathrm{C}$ des étudiants « non communiquant » est décroissant exactement dans l'ordre qui était attendu, avec de bons résultats vite acquis par tous les étudiants pour la production d'exemples localisés (compétence a1) et avec une faiblesse de l'expression (c2) à laquelle on pouvait aussi s'attendre de la part d'étudiants «non communiquant». Ce qui nous a plus surpris, c'est le fort parallélisme des résultats de ce groupe avec ceux du groupe B dont cinq niveaux de progression sont très légèrement inférieurs (ce que nous ne savons pas vraiment expliquer). En revanche, les compétences dans lesquelles les étudiants du groupe B se sont distingués de ceux du groupe $\mathrm{C}$ ne sont pas surprenantes puisqu'elles attestent de leur meilleure maîtrise acquise du vocabulaire géographique (b1) et de l'expression (c2) dont seule la première a pu bénéficier de l'enseignement dans cette discipline. On notera au passage que c'est sur l'aspect lexical (b1) que le niveau global des performances des trois groupes a été le plus élevé alors qu'il n'était pas, a priori, celui sur lequel l'enseignant souhaitait le plus faire porter son effort d'enseignement. Cela s'est peut-être traduit par un biais dans sa notation, plus généreuse sur cet aspect ? Ou alors c'est une rapide adaptation des étudiants qui a entrainé la forte progression de leur compétence en la matière. La question reste sans réponse tranchée.

Les écarts du «profil » du groupe A par rapport aux deux autres profils peuvent être considérés comme des effets directs du travail d'autorégulation des étudiants soutenu par les échanges avec l'enseignant. La différence constatée avec les résultats du groupe B, surtout si ces derniers sont comparables à ceux du groupe $\mathrm{C}$, pourrait être même directement imputée à l'existence d'une procédure de communication renforcée entre étudiants et enseignants plutôt qu'à tout autre facteur. A cet égard, on constate que les plus fortes progressions des résultats du groupe A ont concerné la compétence d'élaboration des introductions (a2) et la compréhension de la spatialité (b2) qui ont, toutes deux, fait l'objet des principaux efforts de l'enseignant, la première surtout au premier semestre et l'autre au second. Pour ces deux compétences, les positions relatives des trois groupes sont exactement les mêmes sur le graphique.

Il reste enfin à commenter les résultats moins probants enregistrés pour les deux dernières compétences qui n'ont pas encore été évoquées et qui occupent des positions équivalentes sur le graphique. La maîtrise des savoirs factuels (c1) était directement dépendante du travail personnel de lecture et de documentation des étudiants. Son évaluation visait juste à indiquer le niveau requis en la matière pour l'épreuve du concours. Elle appelle donc peu de commentaires supplémentaires. En revanche, l'aptitude à structurer un plan par une problématique (compétence d3) nous a semblé difficile à travailler et à «négocier » avec les étudiants car la compétence consistant à construire un plan est survalorisée dans les représentations qu'en ont les étudiants alors qu'ils sous-estiment trop souvent l'intérêt de la problématisation, notamment sous la forme de débats d'idées ou d'opinions en géographie. Cela tient à une conception édulcorée de la géographie qui incite les étudiants à y dénoncer tout au plus les méfaits de la pollution sans y trouver matière à argumentation ou à polémiques. La survalorisation du 
plan, qu'il est difficile de démystifier pour un enseignant qui cherche à encourager le travail personnel de ses étudiants, va dans le sens d'une vision réductrice de la discipline géographique que le contexte des concours nationaux de recrutement n'aide pas à clarifier. Il restera à voir si ces deux compétences donnent lieu à des résultats semblables dans une discipline différemment considérée et jugée plus favorable aux débats d'idées : l'histoire. Une étude complémentaire en cours sur les travaux réalisés par les mêmes étudiants, dans les mêmes conditions avec leur professeur d'histoire, devrait nous aider à préciser ce point.

\section{Conclusion}

Notre étude vise à étudier comment dans un dispositif de formation à distance, un enseignant peut contribuer à développer l'auto direction de ses étudiants. Dans le dispositif analysé, l'enseignant a identifié sept critères de réussite propres à sa discipline et a proposé une communication structurée autour de ces critères. Cette organisation devait permettre à l'étudiant, selon les phases de l'auto direction mises en évidence par Zimmermannn, d'anticiper (l'explicitation des objectifs et des critères d'évaluation favorise l'analyse de la tâche), d'agir (l'étudiant peut utiliser différentes stratégies en hiérarchisant les critères travaillés) et de réfléchir (en communiquant avec l'enseignant et en évaluant les conseils prodigués). Nous avions émis l'hypothèse qu'en identifiant et en explicitant de manière fine les objectifs et les critères de réussite et en développant la communication avec les étudiants, cette démarche aurait pour effet de favoriser leur autonomie de travail et leur progression. Cette hypothèse s'est vérifiée avec les étudiants qui se sont approprié la démarche proposée. Ce sont, en effet, les étudiants « communiquant» qui ont le plus progressé quel que soit leur niveau de départ. Une troisième hypothèse concernait l'acquisition différenciée des compétences. L'étude de cas montre là encore, l'effet de la communication sur les résultats des étudiants.

Le dispositif permettait aux étudiants d'identifier différents sous-objectifs [1] et offrait une liberté de choix [14], ce qui a accru la motivation et a favorisé la réussite des étudiants participants. Cette négociation possible des priorités a généré plus de communication avec ces étudiants, ce qui a favorisé leur auto direction et a renforcé la communication, dans un effet en spirale. Pour déclencher ce cercle vertueux, une stratégie possible pour l'enseignant serait de privilégier en premier les critères les plus accessibles et les plus susceptibles de mettre les étudiants en réussite rapide ce qui augmente la motivation et favorise une communication susceptible de développer ensuite les compétences les plus complexes. Deux questions restent en suspens et nécessitent des travaux ultérieurs : le choix des critères propres à chaque discipline et leurs difficultés supposées croissantes restent soumis à discussion et nécessite d'être testé à plus grande échelle. L'autre question concerne les étudiants les plus fragiles qui globalement communiquent moins que leurs camarades ; mieux identifier, dans ce contexte, les conditions de leur participation demande également de nouvelles recherches.

\section{Références}

[1] Pour une comparaison voir L. Cosnefroy (2010), L'apprentissage autorégulé : perspectives en formation d'adultes. Note de synthèse. Savoirs 23 11-51

[2] A. Jézégou (2008), Apprentissage autodirigé et formation à distance. Distances et savoirs, 6-3, 343-364

[3] B. Zimmermann, Efficacité perçue et autorégulation des apprentissages durant les études : une vision cyclique. In Carré, P. \& Moisan, A. (dir.), (2002), La formation autodirigée, Editions L'Harmattan, coll. Savoir et formation, 69-88

[4] D.R. Garrison (2009), D.R. Communities of Inquiry in Online Learning: Social, Teaching and Cognitive Presence. In C. Howard et al. (Eds.), Encyclopedia of distance and online learning. Hershey, PA : IGI Global 
[5] A. Jézégou (2012), La présence en e-learning : modèle théorique et perspectives pour la recherche. Journal of Distance Education / Revue de l'Education à Distance, 26-1

[6] A. Jézégou (2010), Créer de la présence à distance en e-learning. Cadre théorique, définition, et dimensions clés, Formation à distance, 257-274

[7] P. Bressoux (2002), Contribution à l'analyse de l'effet-maître et des pratiques de classe, in $L a$ gestion de la classe, chapitre 10, De Boeck Supérieur, 199-214

[8] J-J. Quintin (2008), Accompagnement d'une formation asynchrone en groupe restreint : modalités d'intervention et modèles de tutorat, Revue STICEF, 15

[9] D. Mendibil, Les gestes du métier, in M.-C. Robic (dir.) (2006), Couvrir le monde. Un grand XXe siècle de la géographie française, Association pour la Diffusion de la Pensée Française, Ministère des Affaires Etrangères, 54-89

[10] G. Labinal, Les conceptions géographiques du lieu, de l'espace et du territoire, in P. Clerc (dir.), Florence Deprest, G. Labinal, D. Mendibil (2012), Géographies. Epistémologie et histoire des savoirs sur l'espace, chapitre 37, 211-214

[11] M. Fabre, M. Altet, P. Rayou (1998), Hétérogénéité et réussite dans le premier cycle universitaire : enseignants et étudiants, représentations et adaptations des métiers, Rapport de recherche pour le CNCRE

[12] V. Glikman (2002), Des cours par correspondance au e-learning, PUF, $256 \mathrm{p}$

[13] I. Borras (2011), Le tutorat à l'université. Peut-on forcer les étudiants à la réussite ? Bref du Céreq, 290

[14] A. Jézégou (2008), Apprentissage autodirigé et formation à distance, Distances et savoirs, 6-3, $34,3-364$ 